\title{
The prognostic value of estimating stroke volume before and after exercise during treadmill stress echocardiography
}

\author{
Benjamin Fitzgerald ${ }^{1}$, Jelena Logan ${ }^{1}$, Ashleigh Weldon ${ }^{1}$, Agatha Kwon ${ }^{1}$, Isabel Scalia ${ }^{2}$, \\ and Gregory Scalia ${ }^{3}$ \\ ${ }^{1}$ GenesisCare \\ ${ }^{2}$ Royal Brisbane and Woman's Hospital Health Service District \\ ${ }^{3}$ The Prince Charles Hospital
}

July 7, 2020

\begin{abstract}
Background: Stress echocardiography (SE) is an established technique for assessment of coronary artery disease (CAD) which is difficult to perform and interpret. Left ventricular stroke volume (SV) is readily estimated with Doppler echocardiography. It can be affected by myocardial ischemia, with possible adjunctive value during SE. Methods: Patients underwent Bruce protocol SE with SV estimated before and after maximal treadmill exertion post routine regional wall analysis. Incremental change in SV $(\Delta \mathrm{SV})$ with exercise was measured. Results: A derivation cohort $(\mathrm{n}=273)$ was established to test the hypothesis. An optimal cut-off for detection on inducible ischemia was $\Delta \mathrm{SV}[?]+10 \mathrm{ml}$. The validation cohort of consecutive patients ( $\mathrm{n}=1093,376$ [34\%] female; age 59 \pm 12 years) were followed clinically after SE for 20,460 patient-months. There were 1000 patients with non-ischemic SE, and 93 patients with studies suggestive of myocardial ischemia. Secondary analysis yielded 831 patients with a normal exercise response $(\Delta \mathrm{SV}>+10 \mathrm{ml})$ and 192 with an abnormal $\Delta \mathrm{SV}[?]+10 \mathrm{ml}$. Time to first combined adverse cardiac event (composite of angina, acute coronary syndrome, cardiac revascularization, worsening New York Heart Association (NYHA) class, a reduction in EF, and cardiovascular death) was analysed and adjusted using Cox proportional hazards regression. The hazard ratio for an adverse event with an abnormal $\Delta$ SV response ([?]10ml) was 10.3 (95\% confidence intervals 5.6-19.1, $\mathrm{p}<0.0001)$. Conclusions: SV assessment during SE is feasible and readily performed. It is simple, practical and has incremental diagnostic and prognostic value when added to exercise regional wall motion analysis.
\end{abstract}

\section{Introduction}

Stress echocardiography (SE) using treadmill stress has been well validated for the detection of myocardial ischemia. ${ }^{1,2}$ It is recommended for assessment of a wide variety of coronary artery disease (CAD) indications, including for the detection of myocardial ischemia, with a class I recommendation and a level of evidence B. ${ }^{1,2}$ It is "conceptually simple.... However, the simplicity of this concept contrasts with the technical difficulty posed by the practical performance of this technique." 2

Left ventricular stroke volume (SV) and cardiac output (CO) can be readily estimated using echocardiography and have been shown to correlate well with thermodilution calculations in patients. ${ }^{3-5}$ The parameters of SV and $\mathrm{CO}$ can be affected secondary to myocardial ischemia. ${ }^{6,7}$ The estimation of SV with Doppler techniques is straightforward and reproducible. ${ }^{8-10}$ A normal SV response has been shown utilising dobutamine stress echocardiography in patients with no wall motion abnormalities. ${ }^{11}$ This study was designed to assess the incremental value of estimating SV before and after treadmill testing in SE, with subsequent assessment of adverse prognostic cardiac events during patient follow up.

\section{Methods}


Consecutive patients referred to GenesisCare Cardiology clinical testing facilities in Brisbane, Australia were studied prospectively between April 2014 and November 2015. Standard Bruce protocol treadmill testing ${ }^{12}$ with digital gated echocardiography before and after exercise was performed. Patients requiring dobutamine stress echocardiography, patients with resting left bundle branch block, those with a paced rhythm or atrial fibrillation, those with reduced ejection fractions $(\mathrm{EF}<50 \%)$, those with significant valvular regurgitation or stenosis and patients with aortic or mitral valve replacements or mitral valve repairs were excluded. Indications for the test included chest pain and dyspnea for investigation.

The echocardiographic images were acquired in the parasternal long axis, short axis, apical four, two, and three chambers. Ejection fraction was measured by Simpson's method. The SV was estimated by the formula $\mathrm{SV}=$ cross sectional area (CSA) $\mathrm{x}$ velocity time integral (VTI). The CSA of the aortic annulus was calculated using the formula $0.785 \mathrm{x} \mathrm{d}^{2}$, where $\mathrm{d}$ is the left ventricular outflow tract (LVOT) diameter measured in the parasternal long axis plane. The VTI was obtained by measuring the area under the curve of the LVOT pulsed wave Doppler tracing in the apical five chamber view, using the leading edge of the velocity spectrum. ${ }^{8-10}$ (see Figure $1 \& 2$ ) The CO was calculated by multiplying the VTI by the heart rate at the time of the tracing. ${ }^{8-11}$ The resting SV was estimated during the pre-exercise echocardiography scan. The post-exercise value was captured after the peak regional wall assessment, ${ }^{7}$ between 60 and 120 seconds after completion of exercise (see Figure 1). The LVOT diameter was not expected to change post exercise. ${ }^{10}$ The change in stroke volume $(\Delta \mathrm{SV})$ in millilitres $(\mathrm{ml})$ was defined as the SV estimated post exertion minus the resting SV.

Exercise was replicated and quantitated using General Electric medical grade treadmills using Case systems (Milwaukee, USA). Standard Bruce protocols were used to produce exercise stress in a controlled and reproducible environment. Imaging was acquired utilising General Electric Vivid e9 (Horton, USA) and Vivid 7 (Horton, USA), Siemens SC2000 and SC2000 Prime (Mountain View, USA) and the Phillips ie33 and Epic (Best, The Netherlands) echocardiography scanners. Metabolic equivalents (METs) were used to represent exercise capacity, as per standard protocols. ${ }^{1,2,12}$

The echocardiogram was performed by cardiac sonographers with subspecialty training in stress echocardiography. All tests were supervised and read by cardiologists with subspecialty training in stress echocardiography, and an exercise physiologist. Results were then over-read, standardized and recorded by a single stress echocardiography specialised cardiologist, blinded to the results and the outcomes.

Ischemic stress tests were defined as those with new regional wall motion abnormalities in two contiguous segments, or cavity dilatation with a lack of cardiac augmentation (direct comparison of the pre- and post-exercise echocardiographic images). Non-ischemic stress echocardiograms were defined as having no evidence of myocardial ischemia on the stress echocardiogram (no new regional wall motion abnormalities, no cavity dilatation, and appropriate augmentation of cardiac contractility post exercise), with an appropriate augmentation of left ventricular function, as assessed by echocardiography. A wall motion score index $(\text { WMSI })^{1}$ was calculated for all studies by a single stress echocardiography specialised cardiologist, blinded to the results and the outcomes. A subset of patients underwent anatomic testing for coronary obstruction (invasive or CT coronary angiography), as determined by the treating physician, independent of this study.

In order to test the hypothesis that SV could be a marker of abnormal cardiac outcomes, a two-phase study design was devised. An initial cohort of patients was selected and examined (the derivation cohort), to test the hypothesis and if valid to then determine the SV cut-off value. To confirm the concept that the LVOT diameter did not change significantly post exercise, it was measured before and after peak exertion in this derivation cohort. The validation cohort, a subsequent larger group of patients, was then studied to examine the predictive value of an abnormal SV response based on prognostic outcomes.

Patients' medical records were reviewed for up to five (5) years after the baseline SE data collection. Predetermined adverse cardiac endpoints were angina as determined by the patient's clinical cardiologist, acute coronary syndrome, cardiac revascularization (percutaneous intervention or coronary artery bypass grafting, worsening New York Heart Association (NYHA) class, a reduction in EF of greater than 10\%, and cardiovas- 
cular death. A diagnosis of angina, or worsening NYHA class was determined by the treating cardiologist at the time of follow-up. This assessment was made independent to the study, and the treating specialist was blinded to the results. The time interval to a patient's first adverse cardiac event (a composite of the above) was analysed using Cox proportional hazards regression, adjusted for patient's age, sex, ejection fraction, exercise capacity and pre-test Framingham risk, ${ }^{13}$ at the time of the stress echocardiogram.

The study design and methodology were reviewed and approved by the Brisbane GenesisCare Cardiology echocardiographic working group ethics subcommittee. The research protocol was carried out in accordance with The Code of Ethics of the World Medical Association (Declaration of Helsinki). All patients provided written consent.

A multivariable model incorporating the resting and peak change in stroke volume for the prediction of subsequent composite adverse cardiac events in adult patients referred for stress echocardiography was developed. ${ }^{14}$ Data were exported in Microsoft Excel format for subsequent statistical analysis using MedCalc Statistical Software (MedCalc Software, Ostend, Belgium). Demographic and baseline data were tabulated and summarised using descriptive statistics. No imputation for missing data was performed and the number of analysed observations was reported for each summary proportion. Unpaired t-tests were used for cohort analysis of continuous data for separate populations. Paired t-tests were utilized to compare continuous data for dependent variables. The Mann-Whitney test for unpaired samples was used to analyse the median wall motion score index. Data expression was presented as the mean plus or minus the standard deviation.

The outcome for time-to-event analyses within each patient was the first observed element of the study composite outcome of pre-determined endpoints. Results were presented graphically using Kaplan-Meier curves assessed by log-rank tests, as well as tabulated unadjusted and adjusted hazard ratios with 95\% confidence interval (CI) returned by Cox proportional hazard regression models. Variables included in adjusted Cox models were the gender, age in years at the time of baseline assessment, ejection fraction percentage, exercise capacity in METs and pre-test Framingham risk score. The selection of the variables was based on the baseline characteristic differences between groups. The proportional hazards assumption was evaluated using scaled Schoenfeld residuals and visual assessment of log-log plots. The cut-point for SV was determined using receiver operator curves (ROC). ${ }^{14,15}$

\section{Results}

The derivation cohort included 273 consecutive SE with 90 females (33\%) and 183 males. Mean \pm standard deviation (SD) age was $60 \pm 11$ years. The derivation cohort included 250 patients with non-ischemic SE and 23 with tests suggestive of myocardial ischemia. All patients were followed up for a mean $18 \pm 20$ months.

The WMSI was calculated for both groups. The median WMSI for the non-ischemic group was 1 (1-1) and was $1.65(1.50-1.83)$ for the ischemic cohort $(\mathrm{p}<0.0001$ for the difference). The SV was estimated at rest and post exercise and the $\Delta \mathrm{SV}$ calculated. The mean $\Delta \mathrm{SV}$ for the non-ischemic group was $24.0 \mathrm{ml}$ (22.6 to 25.4) compared to $3.4 \mathrm{ml}(-1.1$ to 8.2$), \mathrm{p}<0.0001$ for the difference. A receiver operator curve (ROC) analysis comparing non-ischemic and ischemic SE by $\Delta \mathrm{SV}$ showed that a $\Delta \mathrm{SV}$ of less than $11 \mathrm{ml}$ had an optimal area under the curve (AUC) of 0.95, with a sensitivity of $95 \%$ and a specificity of $99 \%$, p < 0.0001) for myocardial ischemia. See Figure 3. Examples of the Doppler tracings used to estimate the normal and abnormal $\Delta \mathrm{SV}$ values are shown in Figures 1 \& 2 .

Indexing for SV (SV divided by the body surface area) was also performed. The ROC analysis using the $\Delta$ indexed SV $\left(\Delta \mathrm{SV}_{\mathrm{i}}\right)$ resulted in an optimal AUC of 0.78 , with a sensitivity of $72 \%$ and a specificity of $85 \%$, $\mathrm{p}=0.001$ (See Figure 4).

Based on these data, a cut-off value for a "normal $\Delta \mathrm{SV}$ " was set as $>10 \mathrm{ml}$. The $\Delta \mathrm{SV}_{\mathrm{i}}$ was not used, as it appeared to be a less discriminative measure.

To confirm there was no change in the LVOT diameter at the level of the aortic valve before and after exercise, these measurements were taken for all patients in the derivation cohort $(n=273)$. The mean preexercise diameter was $2.25 \mathrm{~cm}$ (95\% CI 2.24-2.27) and was $2.26 \mathrm{~cm}$ (2.24-2.27) post-stress, $\mathrm{p}=0.33$, confirming 
that there was no significant change.

The validation cohort consisted of 1093 consecutive SE that were available for analysis, with 376 females (34\%) and 717 males. Mean \pm standard deviation (SD) age was $59 \pm 12$ years. The baseline characteristics are detailed in Table 1. There were 1000 patients with non-ischemic SE, and 93 patients with SE suggestive of myocardial ischemia. Total follow up was for 20,460 patients-months (up to 5 years per patient, for a mean $37 \pm 23$ months). The SV and CO data for non-ischemic and ischemic stress echocardiograms are listed in Table 2. The estimated SV was able to be performed in approximately $94 \%$ of stress echocardiograms. Limiting factors included post exercise image quality, inadequate Doppler envelopes (with subsequent inadequate VTI measurement) and irregular electrical rhythms, especially bigeminy.

The mean maximum heart rate achieved by patients in the validation cohort was $152 \pm 20 \mathrm{bpm}$, which represented $98 \pm 19 \%$ of the MPHR. The mean heart rate at the time the SV post was acquired was $108 \pm 38 \mathrm{bpm}$, which was $68 \pm 25 \%$ of the maximum predicted heart rate. For the non-ischemic patients, the heart rate was $108 \pm 39$, and for the ischemic patients it was $105 \pm 15, \mathrm{p}=0.19$ (see table 2).

Prognostic events for the follow-up period in the validation cohort were subsequently analysed based on patient SV response to exercise, using the $\Delta \mathrm{SV}$. Utilising the criteria from the derivation cohort, patients were divided into two (2) groups: Group 1 had a normal $\Delta \mathrm{SV}$ response to exercise $(>10 \mathrm{ml})$ and group 2 had an abnormal $\Delta \mathrm{SV}$ response $([?] 10 \mathrm{ml})$. The baseline characteristics of these two groups are detailed in Table 3. The vast majority of the ischemic SE in were in group 2 (98\% of the ischemic SE).

Multivariate Cox proportional hazard regression analysis demonstrated that an abnormal $\Delta \mathrm{SV}$ response $(\Delta \mathrm{SV}[?] 10 \mathrm{ml})$ resulted in a hazard ratio $(\mathrm{HR})$ adjusted for age, sex, exercise capacity and Framingham risk score of 10.3, with $95 \%$ confidence intervals (CI) of 5.6 to 19.1, p<0.0001, for the combined adverse cardiac endpoints, adjusted for age, gender, exercise capacity, ejection fraction and Framingham risk score. The Kaplan Meier curves show early and on-going separation (see Figure 6).

Cardiac deaths were rare events during follow up. None were seen in the normal $\Delta \mathrm{SV}$ group and three (3) noted in the abnormal $\Delta \mathrm{SV}$ cohort. Analysis of these limited data suggested a trend to increased mortality in the abnormal $\Delta \mathrm{SV}$ group with a HR of 12.4 (95\% CI 0.85 to 18.0$), \mathrm{p}=0.066$.

The HR for subsequent invasive catherization based on a $\Delta \mathrm{SV}[?] 10 \mathrm{ml}$ was 4.92 (95\% CI 3.76 to 6.45$)$ $\mathrm{p}<0.0001$, noting that the overwhelming majority of these patients had an ischemic SE. These patients were more likely to proceed to invasive catheterization on a clinical basis, due to the SE result.

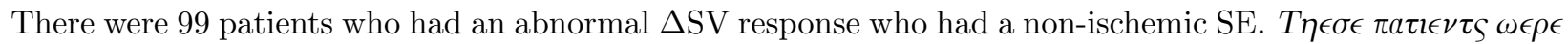

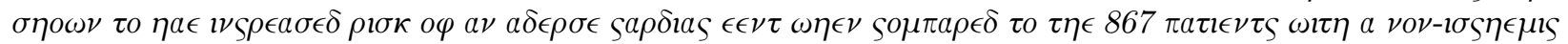

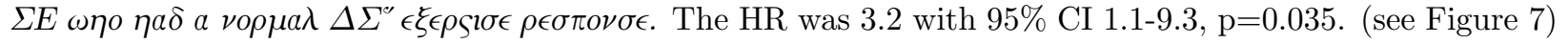
The majority of events appeared to be ischemic in nature (angina pectoris or need for percutaneous coronary intervention). Using a lack of adverse cardiac outcomes as the baseline for a true negative test, the overall negative predictive value for a normal SV response $(\Delta \mathrm{SV}>10 \mathrm{ml})$ was $94 \%$.

\section{Discussion}

Stress echocardiography has become an established and mature technique for the assessment of CAD. ${ }^{1}$ Interpretation remains challenging, even with experienced practitioners. ${ }^{1,2}$ Additional parameters can be utilised to assist with this difficult interpretation, including strain and strain rate, ${ }^{16,17}$ and assessments of diastolic function. ${ }^{1,18}$ None of these have become routine practice.

Left ventricular SV is the amount of blood ejected with each heartbeat. It is the difference between the end-diastolic volume and end systolic volume. ${ }^{11,19-20}$ Exercise results in an increase in cardiac contractility. The changes in the mechanical properties of contracting cardiac muscle fibers with changes in inotropy results in an increase in SV. ${ }^{11,19-20}$ A decrease in cardiac contractility causes a downward shift in the Frank-Starling curve, with subsequent drop in SV (and an increase in left ventricular end diastolic pressure). 
11,19-20 Myocardial ischemia results in changes in contractile state of the ventricle. These changes result in alterations to SV and CO. ${ }^{11,20-21}$

Mechanistically, active human systems require a constant blood supply with delivery of oxygen and nutrients and removal of waste products to function normally. Exercise increases these requirements. Any alteration in supply (e.g. inducible myocardial ischemia) reduces provision of these essential roles, resulting in decreased function. At a ventricular level this results in a drop in SV (and CO). ${ }^{11,19-21}$ These alterations are due to changes in systolic (via a loss of intrinsic inotropy) and diastolic (loss of compliance) function. Left ventricular contractility has been shown to change similarly to SV. ${ }^{20,22}$ At a basic science level, nitric oxide inhibition at an endothelial level results in hemodynamic changes. ${ }^{24,25}$

Echocardiography can reliably estimate SV and $\mathrm{CO}$, before and after stress testing. (see Materials and Methods). ${ }^{7,11,18,26,27}$ The changes in SV and measures of diastolic function have been shown to be reliable when compared to invasive techniques. ${ }^{18,28-31}$ Changes in Doppler echocardiography have been documented in the setting of CAD. ${ }^{32-34}$ These concepts contributed to the hypothesis that SV could be a simple and readily measurable parameter to assist with analysis for stress echocardiography.

This study was designed prospectively to measure SV before and after treadmill exercise, and then to subsequently look at adverse cardiac outcomes in follow-up, as a method of validation. Estimation of the SV before and after exertion provided additional, complimentary and confirmatory information when added to the traditional stress echocardiogram analysis. The estimated SV was used for analysis, as it was a purer and simpler value (there was significantly more variation with the CO estimations, due to marked variability between patients with respect to heart rate, at rest and after stress). Indexing SV resulted in a lower AUC and lower sensitivity and specificity. As such SV was preferred to $\mathrm{SV}_{\mathrm{i}}$. A significant increase in SV with exertion is a normal response. An inadequate response was shown to be either a decrease or a mild increase $(<10 \mathrm{ml})$. This suboptimal SV increment with exertion resulted in significantly worse cardiac prognosis compared to patients who had an appropriate increase in these volumes.

It is proposed that this suboptimal SV change is a marker of ischemia and the increase in events were likely to be driven by this. Its negative predictive value is very high, adding reassurance to negative studies. An abnormal result associated with a non-ischemic SE may prompt consideration for a more careful analysis of the regional wall motion analysis, or further clinical evaluation. The SV analysis appears to provide statistically incremental analytical and prognostic information to the standard SE evaluation.

An interesting subset of patients were those with a non-ischemic SE, but an abnormal $\Delta \mathrm{SV}$. These patients had increased risk compared to non-ischemic patients with a normal $\Delta \mathrm{SV}$ response to exertion, even following adjustment for age, gender, EF, exercise capacity and Framingham risk (see Figure 5). Careful re-evaluation of the SE did not reveal post exertion regional wall motion abnormalities or reduced cardiac augmentation. Looking at the individual cases, the events for this cohort appeared to be ischemic in nature. Possible reasons for an abnormal $\Delta \mathrm{SV}$ despite a non-ischemic SE could include a false negative $\mathrm{SE}$, endothelial dysfunction, subclinical ischemia, inducible diastolic dysfunction (except that $\mathrm{E} / \mathrm{e}^{\prime}$ did not universally correspond with these abnormal $\Delta \mathrm{SV}$ cases), exercise induced pulmonary hypertension, or by chance. These patients did not have increased wall thickness on echocardiography compared to those with a normal $\Delta \mathrm{SV}$. These changes may represent myocardial alterations that are too subtle for visual detection. As the events in this subset appear to be ischemic, this may the driver and the most likely explanation. This finding suggests an incremental value for assessing $\Delta \mathrm{SV}$ in $\mathrm{SE}$.

This technique is quick and readily performed and has been shown here to be valid when measured after the routine regional wall motion analysis. It can be performed in the vast majority of patients. It requires no additional equipment or preparation. It adds only seconds to the resting echocardiogram and to the post exercise analysis and can be done after regional wall analysis but before the estimations of diastolic function. ${ }^{18}$ The proposed cut-off appears to apply for the detection of ischemia and for the detection of adverse cardiac events.

Previous studies have looked at using LV volumes as a marker of coronary artery disease. ${ }^{35,36}$ These stud- 
ies used planimetry and the Simpson's biplane method. This volume measurement essentially documents a change in EF. This method is more time consuming than the Doppler technique and has limitations including the geometrical assumptions used to assess LV function, alignment issues (particularly a problem during exercise stress echocardiography), frequent foreshortening of the ventricle, and the influence of load-dependent factors during functional assessment. ${ }^{10,37}$ Suboptimal endocardial definition further reduces the accuracy of this technique. Regional wall motion abnormalities make dynamic assessment even more challenging. ${ }^{10,37}$ The Doppler estimation of SV measures a change in ventricular volume utilising a different technique which has been shown here to be independent of EF. This Doppler technique has previously been shown to be feasible in dobutamine stress echocardiography to estimate $\mathrm{CO}{ }^{11}$ but has not been used in the manner described in this study.

There are limitations with respect to this observational cohort. Ideally these measurements would have been compared to invasive measurements to confirm the diagnosis and measure SV invasively, blindly. Practically, ethically and from the clinical perspective, this was not feasible and would have negated the non-invasive attribute of stress echocardiography. The ethical concerns refer to universally invasively testing low to intermediate risk patients. ${ }^{19,38-40}$ The accuracy of SE for detection of myocardial ischemia has already been previously documented and validated. ${ }^{1,2,39,40}$ In general, stress echocardiography is used to attempt to avoid an invasive test. Clinically, a select group of patients (as determined by the treating physician, and independent and blinded to the study) had an anatomical evaluation, which confirmed the efficacy and accuracy of SE. The median WMSI values were significantly different for non-ischemic patients in both the derivation and validation cohorts.

Baseline clinical characteristics were documented but blood tests were not performed. This was done to simplify the study and minimise the intrusion on the volunteers. Baseline bloods potentially could have added further data to the interpretation of these results.

Inconsistency in the measurement of SV may lead to a lack of reproducibility. Significant variability in the $\mathrm{SV}$ measurement could compromise the cut-off values. However, the difference between the mean $\Delta \mathrm{SV}$ and the cut-off values were marked (see Table 2) and unlikely to fall within the margin of error of acquisition. The estimation of SV requires an accurate measurement of the left ventricular outflow tract. A small error in this measurement magnifies the error. ${ }^{10,37}$ Previous studies have suggested that the echocardiographic measurement of SV is an accurate and reproducible technique. ${ }^{3-5,44}$ In order to minimise inter-reader variability, a single experienced, echocardiography subspecialty cardiologist measured all the stroke volume data, blinded to the outcomes. This may reduce the applicability of these data to the wider community but minimised errors.

Whilst patients achieved a mean $98 \pm 19 \%$ of maximum predicted heart rate at peak exercise, by the time the SV was acquired, this value had reduced to $68 \pm 25 \%$, well below the value recommended to maximise sensitivity for detection of ischemia. ${ }^{38,45,46}$ Despite this, abnormal $\Delta$ SV values predicted prognostic differences, suggesting that delaying this measurement until after the regional wall motion analysis still provides valid and valuable additional information to the standard test method.

It is possible that the results were simply due to the presence or absence of ischemia, or reflective of the changes in ejection fraction post exertion. However, patients with a normal $\Delta \mathrm{SV}$ were seen in the ischemic group, and an abnormal $\Delta \mathrm{SV}$ was seen in patients with a non-ischemic SE. All patients with a non-ischemic $\mathrm{SE}$ had a normal increase in EF post exertion. The data presented here suggest that $\Delta \mathrm{SV}$ is a more sensitive marker of events than EF or a normal SE response.

The estimation of SV cannot be performed in all patients. Heart rate did not produce any issues, but peak exercise image quality occasionally limited the completeness of the Doppler envelope, resulting in inadequate measurements. In most cases, image quality had less of an impact on the ability to estimate Doppler signals. Irregular electrical rhythms, especially bigeminy resulted in Doppler signals that were difficult to accurately measure. These incomplete measurements only occurred in a very small percentage of patients. Stroke volume could be successfully compared pre and post exercise in approximately $94 \%$ of these stress 
echocardiograms.

In this non-randomized, single center cohort study, there were some apparent differences between the groups at baseline (see Table 1). Despite presentation of the adjusted estimates, these baseline differences may have influenced the outcomes presented here.

The evaluation of the medical records was a potential source of ascertainment bias. It is possible for events to occur at other centres, and potentially not be recorded in these patient reviews. The ischemic patients may have been assessed more closely due to the results of the stress test, resulting in a higher reporting of events. However, given these patients had significantly more abnormal $\Delta \mathrm{SV}$ measurements, an under-detection of events in this group would not be expected to influence these results. The SV analysis was conducted after events had occurred, and the results were not communicated to the clinicians, as the implications were not apparent at the time of data collection. While failure to appropriately account for missing data in analyses may lead to bias and loss of precision, imputation of missing results also requires additional assumptions. To have completed this analysis with complete data rather than imputation of missing values may not have been associated in an epidemiological context with substantial bias in reported regression estimates. ${ }^{15,41}$

The multivariable prediction model described in the present study was derived from echocardiographic observations at a single center. There were significantly less women than men (a common problem in cardiac research). This does reflect real world experiences and this particular study population. 18,42,43 The investigators were not blinded to the results of the stress test, making it possible for biases (including ascertainment bias) to occur. Overall event rates were low, especially in the non-ischemic patients. The present model would benefit from validation within an independently collected data set from a separate population.

The broad referral pattern of these patients does suggest that the utility of measuring SV could be extended to the general stress test population.

\section{Conclusions}

Stress echocardiography is a well-established technique for the detection of myocardial ischemia but is very technically difficult to perform and interpret. An estimation of stroke volume before and after treadmill exercise is feasible, quick and readily performed. In the setting of adequate augmentation of SV ( $>10 \mathrm{ml})$, the negative predictive value for detection of ischemia and adverse cardiac events is very high, improving the diagnostic value of SE as a rule out test. It has practical and prognostic incremental value in the setting of ischemic and non-ischemic tests. These data show that this remarkably simple technique is a very powerful predictor of outcome. It is biologically plausible. It would appear to provide a new benchmark for the noninvasive detection and exclusion of ischemia. Adding stroke volume measurements before and after exertion can improve outcome prediction for stress echocardiography.

The authors attest that they are in compliance with human studies committees of the authors' institutions and international guidelines including patient consent. No animals were used as part of this research.

This research did not receive any specific grant from funding agencies in the public, commercial, or not-forprofit sectors.

Conflict of interest: None

\section{Abbreviation list :}

AUC - Area under the curve

CAD -Coronary artery disease

CI - Confidence intervals

$\mathrm{CO}$ - Cardiac output

CSA - Cross sectional area 
CT - Computer tomographic

$\Delta \mathrm{SV}$ - Delta stroke volume

$\mathrm{EF}-$ Ejection fraction

HR - Heart rate

LVOT - Left ventricular outflow tract

METs - Metabolic equivalents

MPHR - The age derived Maximum Predicted Heart Rate

NYHA - New York Heart Association

ROC - Receiver operator curve

SD - Standard deviation

SE - Stress echocardiogram

$\mathrm{SV}$ - Stroke volume

$\mathrm{SV}_{\mathrm{i}}$ - Indexed stroke volume

VTI - Velocity time integral

WMSI - Wall motion score index

\section{Author contributions}

Dr Fitzgerald is the lead author and devised the concept and design, created the database, analysed, and interpreted the data, and drafted and revised the paper. Professor Scalia was involved in the design, drafting and revision and created some of the complex graphics utilised. Ms Logan, Ms Weldon and Ms Kwon had roles in perfecting the design, collecting, analysing and interpreting the data and in the manuscript revision. Dr Scalia was involved in collection, analysis, and interpretation of the data, and in drafting and revising the document. All authors have reviewed and approved the manuscript.

\section{References}

1. Pellikka PA, Arruda-Olson A, Chaudhry FA, et al. Guidelines for Performance, Interpretation, and Application of Stress Echocardiography in Ischemic Heart Disease: From the American Society of Echocardiography. J Am Soc Echocardiogr. 2020; 33(1):1-48.

2. Marwick TH. Stress Echocardiography - Its Role in the Diagnosis and Evaluation of Coronary Artery Disease. 2 ed. Boston: Kluwer Academic Publishers; 2003: 1-93

3. Gueret P, Meerbaum S, Zwehl W, et al. Two-dimensional echocardiographic assessment of left ventricular stroke volume: experimental correlation with thermodilution and cineangiography in normal and ischemic states. Cathet Cardiovasc Diagn. 1981;7(3):247-58

4. Limacher MC, Quinones MA, Poliner LR, et al. Detection of coronary artery disease with exercise two-dimensional echocardiography. Description of a clinically applicable method and comparison with radionuclide ventriculography. Circulation. 1983 Jun;67(6):1211-8.

5. Christie J, Sheldahl LM, Tristani FE, et al. Determination of stroke volume and cardiac output during exercise: comparison of two-dimensional and Doppler echocardiography, Fick oximetry, and thermodilution. Circulation. 1987;76(3):539-47.

6. Elhendy A, Mahoney DW, Khandheria BK, et al. Prognostic significance of impairment of heart rate response to exercise: impact of left ventricular function and myocardial ischemia. J Am Coll Cardiol. 2003;42(5):823-30. 
7. Supino PG, Herrold EM, Braegelman F, Borer JS. Left ventricular ejection fraction change with exercise versus ejection fraction at rest in coronary artery disease: implications for using ejection fraction variations in making therapeutic decisions. Am J Ther. 2004;11(3):164-70.

8. Anderson BA. Echocardiography: The Normal Examination and Echocardiographic Measurements. $3^{\text {rd }}$ ed. Brisbane, Echotext, 2017:186-8.

9. Gardin JM, Tobis JM, Dabestani A, et al. Superiority of two-dimensional measurement of aortic vessel diameter in Doppler echocardiographic estimates of left ventricular stroke volume. J Am Coll Cardiol. 1985;6(1):66-74.

10. Otto CM. Textbook of Clinical Echocardiography. $2^{\text {nd }}$ Edition. Philadelphia, W.B Saunders, 2000:3337, 51-56, 232-243.

11. Pellikka PA, Roger VL, McCully RB, et al. Normal stroke volume and cardiac output response during dobutamine stress echocardiography in subjects without left ventricular wall motion abnormalities. Am J Card. 1995; 12: 881-6.

12. Bruce RA, McDonough JR. Stress testing in screening for cardiovascular disease. Bull N Y Acad Med. 1969;45(12):1288-305.

13. D'Agostino RB Sr, Vasan RS, Pencina MJ, et al. General cardiovascular risk profile for use in primary care: the Framingham Heart Study. Circulation. 2008;117(6):743-53.

14. Moons KG, Altman DG, Reitsma JB, et al. Transparent Reporting of a multivariable prediction model for Individual Prognosis or Diagnosis (TRIPOD): explanation and elaboration. Ann Intern Med. 2015;162(1):W1-73.

15. Grambsch PM, Therneau TM. Proportional hazards tests and diagnostics based on weighted residuals. Biometrika. 1994;81:515-26.

16. Bjork Ingul C, Rozis E, Slordahl SA, Marwick TH. Incremental value of strain rate imaging to wall motion analysis for prediction of outcome in patients undergoing dobutamine stress echocardiography. Circulation. 2007;115(10):1252-9.

17. Takemoto Y, Pellikka PA, Wang J, et al. Analysis of the interaction between segmental relaxation patterns and global diastolic function by strain echocardiography. J Am Soc Echocardiogr. 2005;18(9):9016.

18. Fitzgerald BT, Presneill JJ, Scalia IG, et al. The Prognostic Value of the Diastolic Stress Test in Patients Undergoing Treadmill Stress Echocardiography. J Am Soc Echocardiogr. 2019 Oct;32(10):12981306.

19. Mann D, Zipes D, Libby P, Bonow R. Braunwald's Heart Disease: A Textbook of Cardiovascular Medicine. 10 ed. 10 ed. Philadelphia: Saunders; 2015:479-500

20. Laughlin MH, Cardiovascular Response to exercise. Am J Physiol. 1999; 277: S244-259.

21. Bogen DK, Needleman A, McMahon TA. An analysis of myocardial infarction. The effect of regional changes in contractility. Circ Res. 1984 Dec;55(6):805-15.

22. Lujan HL, DiCarlo SE. Cardiac output, at rest and during exercise, before and during myocardial ischemia, reperfusion, and infarction in conscious mice. Am J Physiol Regul Integr Comp Physiol. 2013; 304(4): R286-95

23. Huber W, Zanner R, Schneider G, et al. Assessment of Regional Perfusion and Organ Function: Less and Non-invasive Techniques. Front Med (Lausanne). 2019;6:50. doi: 10.3389/fmed.2019.00050.

24. Arbab-Zadeh A, Dijk E, Prasad A, et al. Effect of Aging and Physical Activity on Left Ventricular Compliance. Circulation. 2004; 110:1799-1805.

25. Gaballa MA, Raya TE, Hoover CA, Goldman S. Effects of endothelial and inducible nitric oxide synthases inhibition on circulatory function in rats after myocardial infarction. Cardiovascular Research, 1999;42 (3):627-635.

26. Thijssen DHJ, Maiorana AJ, O'Driscoll G, et al. Impact of inactivity and exercise on the vasculature in humans. Eur J Appl Physiol. 2010;108(5): 845-875.

27. Daley PJ, Sagar KB, Collier BD, et al. Detection of exercise induced changes in left ventricular performance by Doppler echocardiography. Br Heart J. 1987 Nov;58(5):447-54.

28. Ihlen H, Endresen K, Golf S, Nitter-Hauge S. Cardiac stroke volume during exercise measured by 
Doppler echocardiography: comparison with the thermodilution technique and evaluation of reproducibility. Br Heart J. 1987;58(5):455-9.

29. Sweet RL, Moraski RE, Russell RO Jr, Rackley CE. Relationship between echocardiography, cardiac output, and abnormally contracting segments in patients with ischemic heart disease. Circulation. 1975;52(4):634-41.

30. Gardin JM, Tobis JM, Dabestani A, et al. Superiority of two-dimensional measurement of aortic vessel diameter in Doppler echocardiographic estimates of left ventricular stroke volume. J Am Coll Cardiol. 1985;6(1):66-74.

31. Ihlen H, Myhre E, Pamlie J, et al. Changes in left ventricular stroke volume measured by Doppler echocardiography. Br Heart J. 1985;54(4):378-83.

32. Ihlen H, Endresen K, Myreng Y, Myhre E. Reproducibility of cardiac stroke volume estimated by Doppler echocardiography. Am J Cardiol. 1987;59(9):975-8.

33. Masuyama T, Kodama K, Nakatani S, et al. Effects of changes in coronary stenosis on left ventricular diastolic filling assessed with pulsed Doppler echocardiography. J Am Coll Cardiol. 1988 Apr;11(4):74451.

34. Stoddard MF, Pearson AC, Kern MJ, et al. Left ventricular diastolic function: comparison of pulsed Doppler echocardiographic and hemodynamic indexes in subjects with and without coronary artery disease. J Am Coll Cardiol. 1989;13(2):327-36.

35. Olson CE, Porter TR, Deligonul U, Xie F, Anderson JR. Left Ventricular Volume Changes During Dobutamine Stress Echocardiography Identify Patients With More Extensive Coronary Artery Disease. J Am Coll Cardiol. 1994; 24(5):1268-73.

36. C Coletta C, Galati A, Ricci R, et al. Prognostic Value of Left Ventricular Volume Response During Dobutamine Stress Echocardiography. Eur Heart J. 1997; 18(10): 1599-605.

37. Luis S.A, Chan J, Pellikka P. Echocardiographic Assessment of Left Ventricular Systolic Function: An Overview of Contemporary Techniques, Including Speckle-Tracking Echocardiography. Mayo Clinic Proceedings. 2019; 94(1):125-138.

38. Marwick TH. Stress Echocardiography. In:Topol E. Textbook of Cardiovascular Medicine. 2nd ed. Philadelphia: Lippincott Williams \& Wilkins; 2002:1267-1300.

39. Do D, West JA, Morise A, et al. An agreement approach to predict severe angiographic coronary artery disease with clinical and exercise test data. Am Heart J. 1997;134:672-9.

40. Schiano-Lomoriello V, Santoro C, de Simone G, et al. Diastolic bicycle stress echocardiography: Normal reference values in a middle age population. Int J Cardiol. 2015;191:181-3.

41. Hughes RA, Heron J, Sterne JAC, Tilling K. Accounting for missing data in statistical analyses: multiple imputation is not always the answer. Int J Epidemiol. 2019, Mar 16. doi: 10.1093/ije/dyz032.

42. Fitzgerald BT, Scalia WM, Scalia GM; Female False Positive Exercise Stress ECG Testing - Fact Versus Fiction. Heart, Lung and Circulation, 2019;28:735-741

43. Fitzgerald BT, Ballard EL, Scalia GM; Estimation of the Blood Pressure Response with Exercise Stress Testing. Heart, Lung and Circulation, 2019;28:742-51

44. Yajima T, Nitta M, Nakamura S, et al. Reliability of Doppler Echocardiographic Stroke Volume Measurement. Bull Tokyo Med Dent Univ. 1992; 39(3):55-62.

45. Marwick TM, Nemec JJ, Pashkow FJ, et al. Accuracy and limitations of exercise echocardiography in a routine clinical setting. J Am Coll Cardiol. 1992; 19(1):74-81.

46. Cumming GR. Yield of ischaemic exercise electrocardiograms in relation to exercise intensity in a normal population. Br Heart J; 1972;34(9):919-23

\section{Figure Legends:}

Figure 1. LVOT pulsed wave Doppler tracing at rest and post peak exercise, used to calculate the normal $\Delta \mathrm{SV}$ response to exercise and subsequent estimation of VTI.

Figure 2. LVOT pulsed wave Doppler tracing at rest and post peak exertion, used to calculate the abnormal $\Delta \mathrm{SV}$ response to exercise and subsequent estimation of VTI. 
Figure 3. ROC analysis for the derivation cohort to determine the cut-off value for $\Delta \mathrm{SV}$.

Figure 4. ROC analysis showing that $\Delta \mathrm{SV}$ is more discriminative than $\Delta \mathrm{SV}_{\mathrm{i}}$ and to determine the cut-off value for $\Delta \mathrm{SV}$

Figure 5. Histogram of the distribution of $\Delta \mathrm{SV}$ for the Ischemic and Non-Ischemic patients in the validation cohort.

Figure 6. Kaplan Meier curves for the combined cardiac adverse event by Stroke Volume response to exercise in the validation cohort

Figure 7. Kaplan Meier curves for the combined cardiac adverse event by Stroke Volume response to exercise for all non-ischemic stress echocardiograms in the validation cohort $(n=1000)$

\section{Tables.}

Table 1. Baseline characteristics for All patients and for patients with non-ischemic and ischemic stress echocardiograms $(\mathrm{n}=1093)$

Table 2. Stroke volume and cardiac output values for patients with non-ischemic versus ischemic stress echocardiograms $(\mathrm{n}=1093)$

Table 3. Baseline characteristics for patients with a normal $\Delta \mathrm{SV}$ versus an abnormal $\Delta \mathrm{SV}(\Delta \mathrm{SV}[?] 10)$

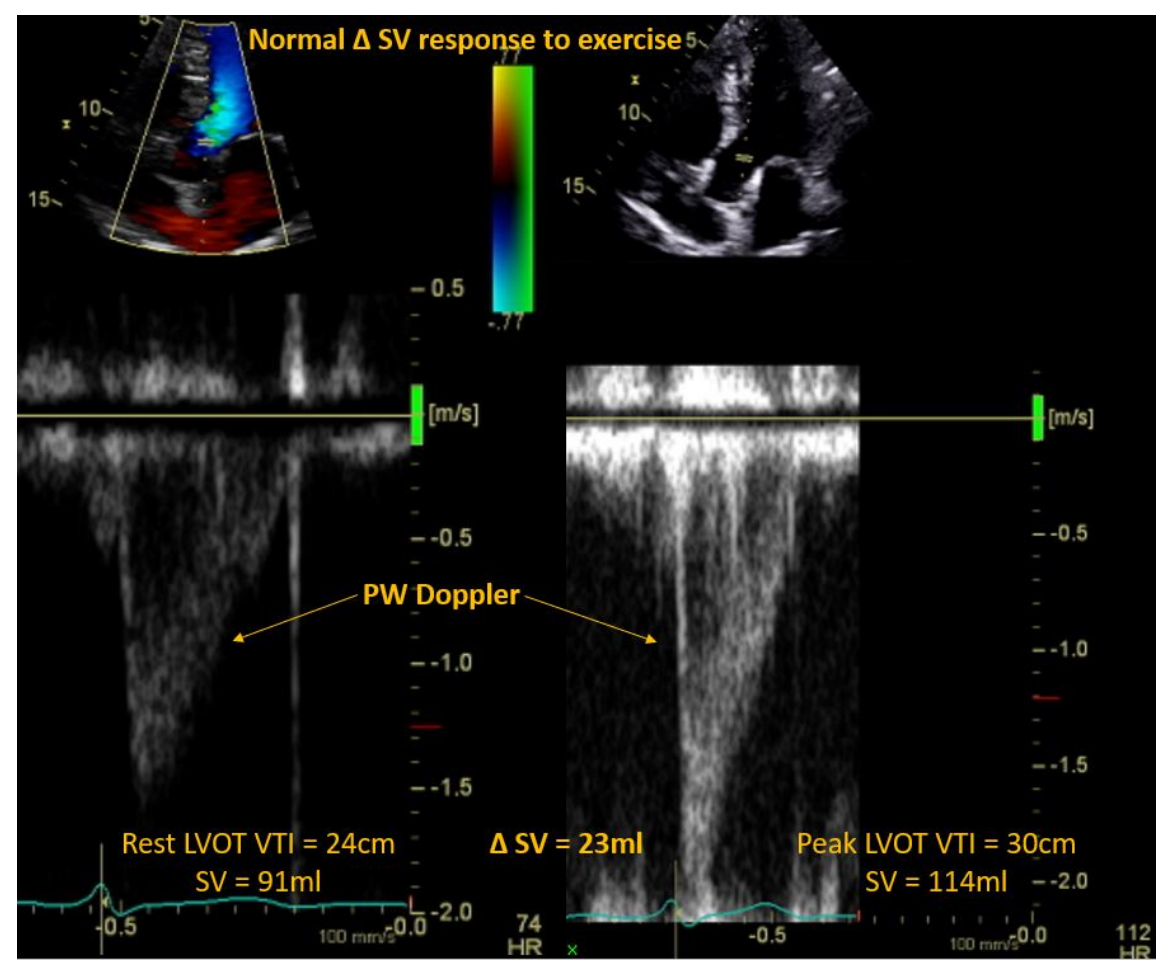




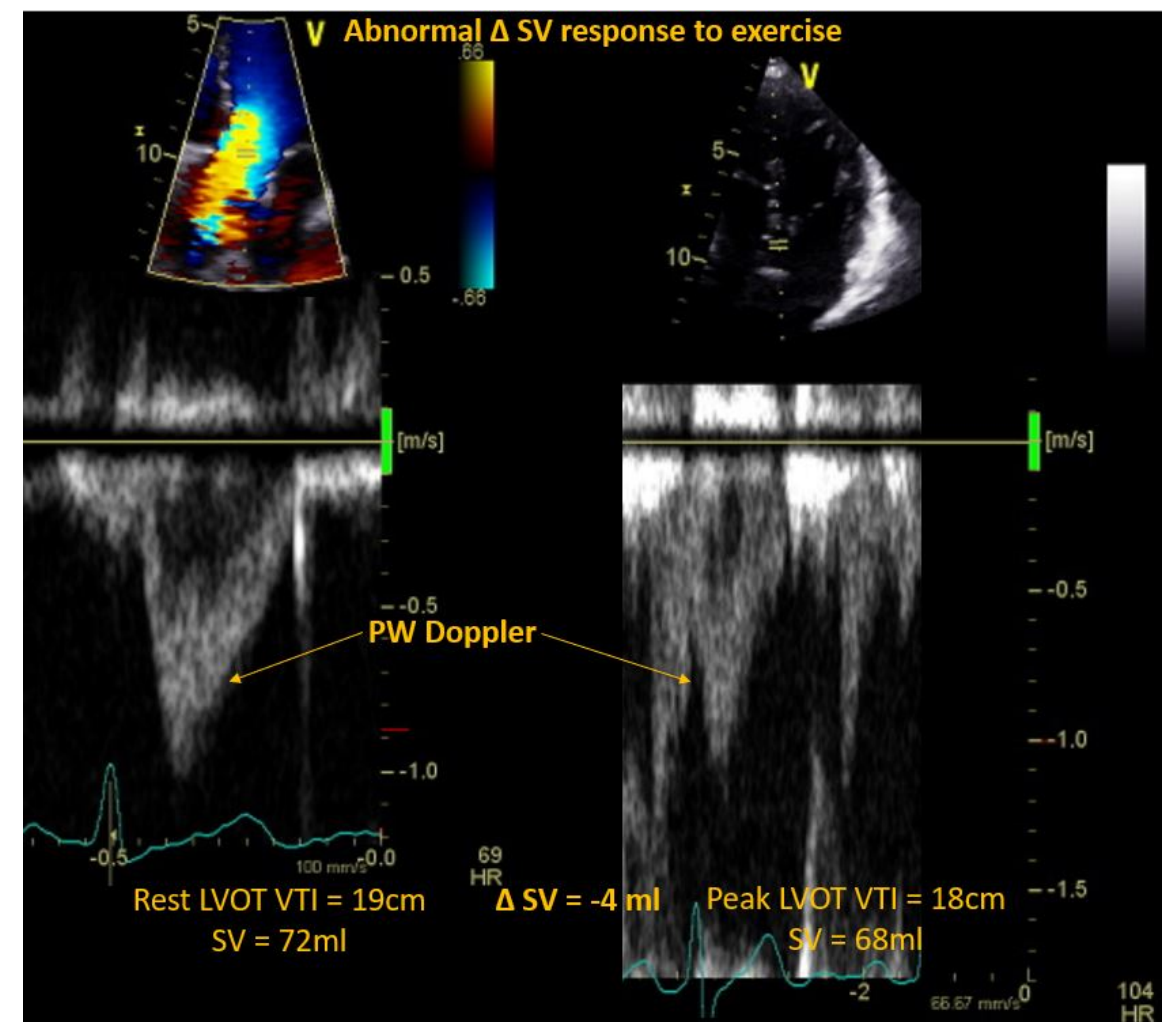


Figure 3. ROC analysis for the derivation cohort to determine the cut-off value for $\Delta S$ V.

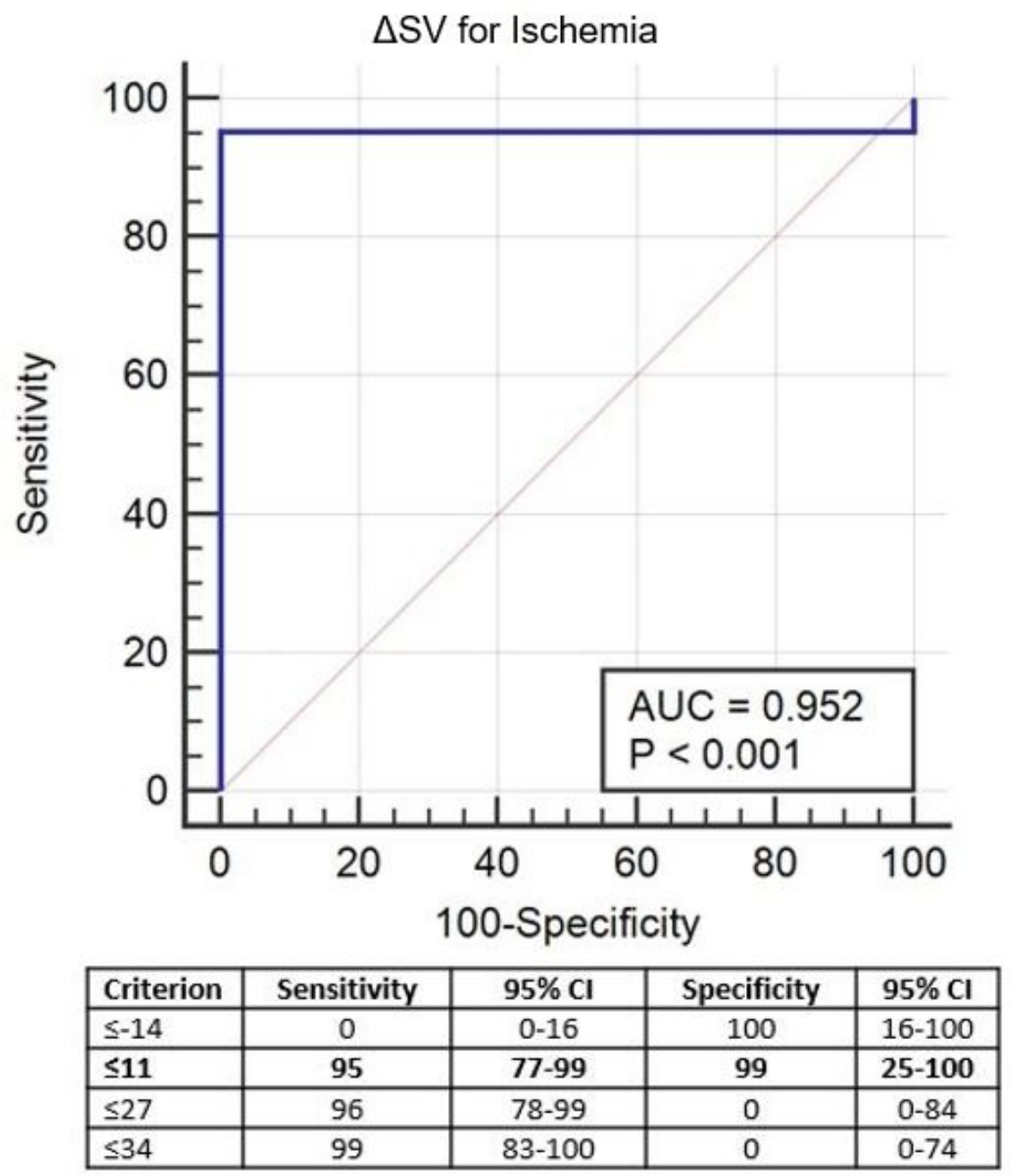


Figure 4. ROC analysis showing that $\triangle \mathrm{SV}$ is more discriminative than $\Delta \mathrm{SV}_{\mathrm{i}}$ and to determine the cut-off value for $\Delta \mathrm{SV}$.
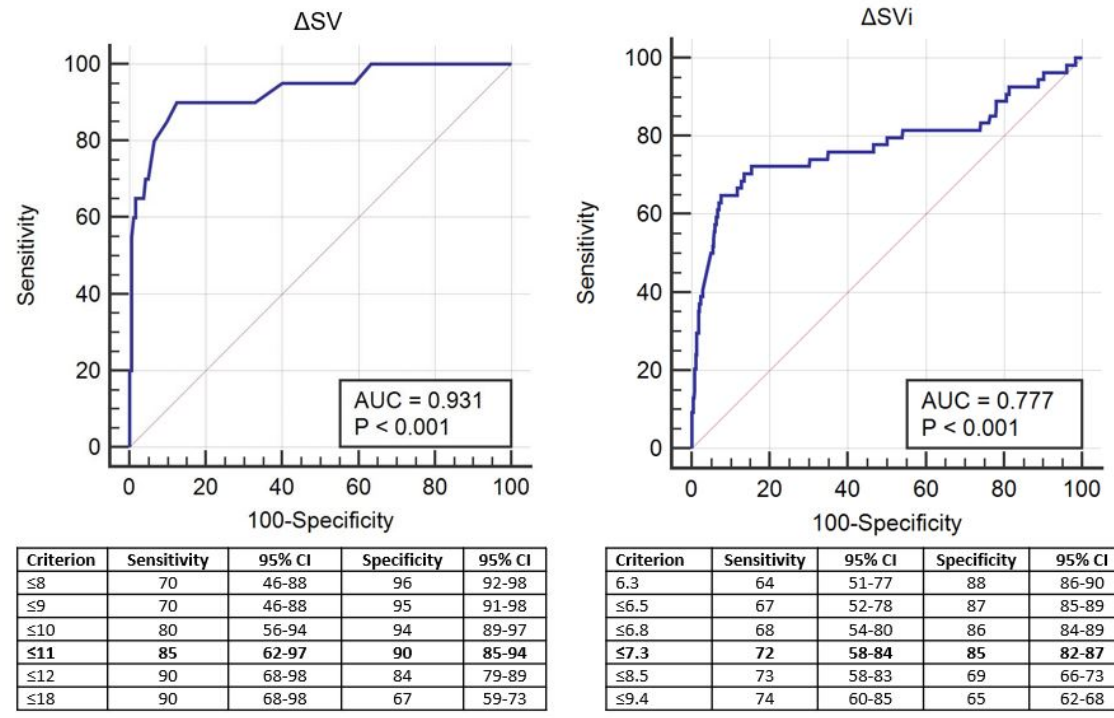

\begin{tabular}{|l|c|c|c|c|}
\hline Criterion & Sensitivity & $\mathbf{9 5 \%} \mathrm{Cl}$ & Specificity & $\mathbf{9 5 \%} \mathrm{Cl}$ \\
\hline 6.3 & 64 & $51-77$ & 88 & $86-90$ \\
\hline$\leq 6.5$ & 67 & $52-78$ & 87 & $85-89$ \\
\hline$\leq 6.8$ & 68 & $54-80$ & 86 & $84-89$ \\
\hline$\leq 7.3$ & $\mathbf{7 2}$ & $\mathbf{5 8 - 8 4}$ & $\mathbf{8 5}$ & $\mathbf{8 2 - 8 7}$ \\
\hline$\leq 8.5$ & 73 & $58-83$ & 69 & $66-73$ \\
\hline$\leq 9.4$ & 74 & $60-85$ & 65 & $62-68$ \\
\hline
\end{tabular}

Figure 5. Histogram of the distribution of $\Delta \mathrm{SV}$ for the Ischemic and Non-Ischemic patients in the validation cohort

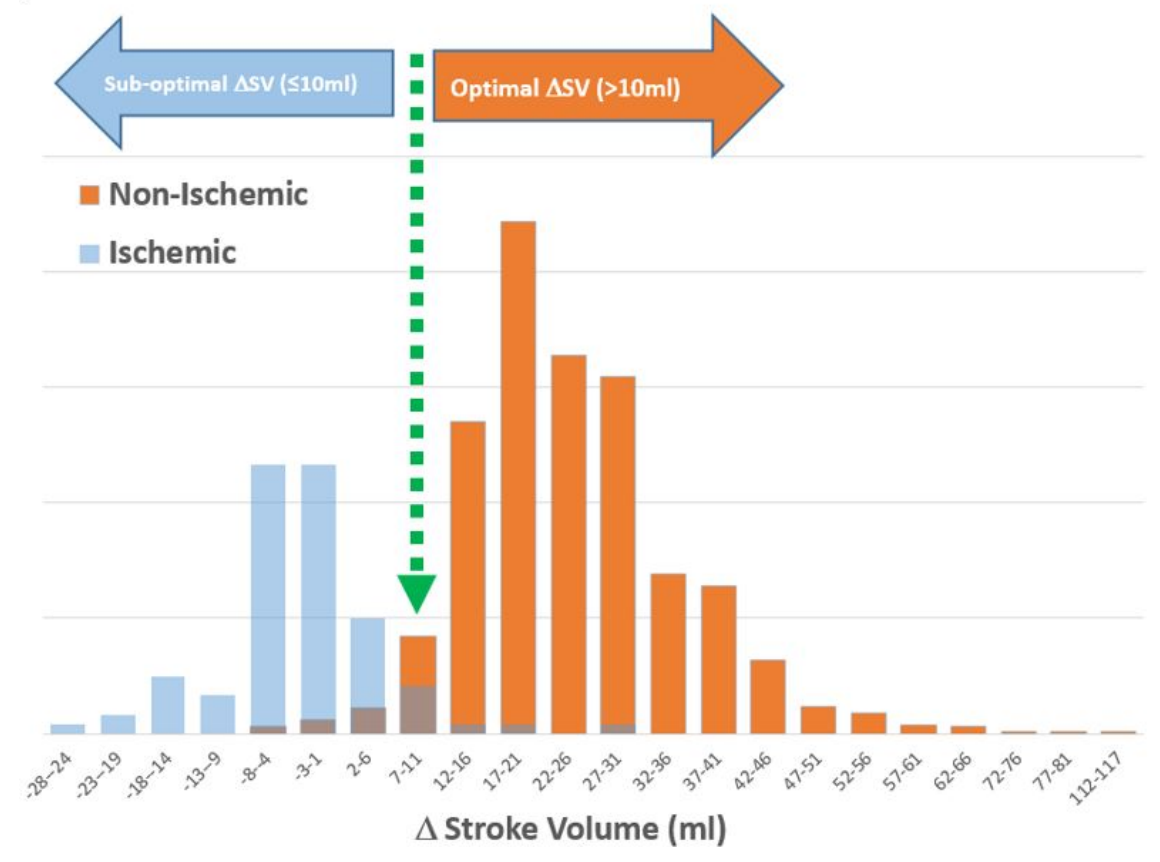


Figure 6. Kaplan Meier curves for the combined cardiac adverse event by SV response to exercise in the validation cohort.

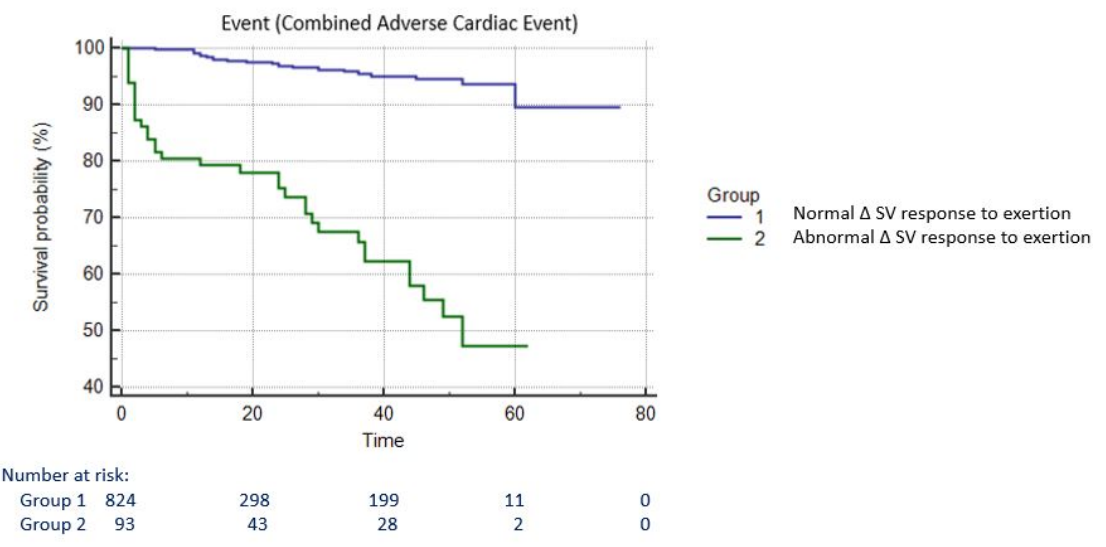

$\Delta S V$ - Delta stroke volume

Figure 7. Kaplan Meier curves for the combined cardiac adverse event by SV response to exercise for all non-ischemic stress echocardiograms in the validation cohort $(n=1000)$.

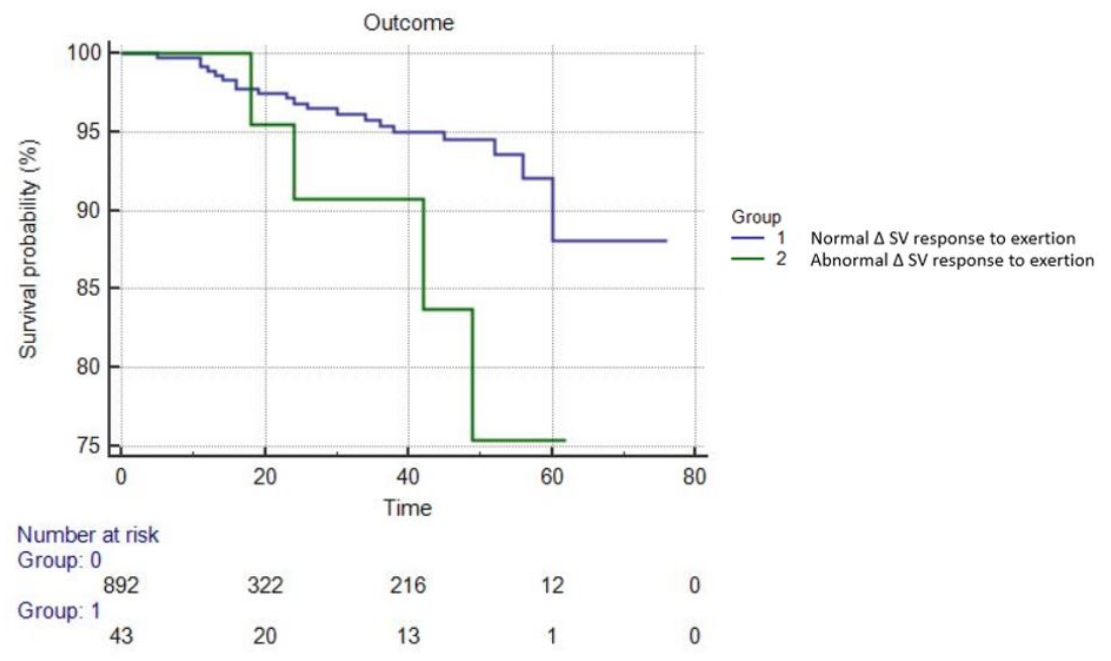

$\Delta \mathrm{SV}$ - Delta stroke volume 